\title{
Effects of Rice Varieties on Weight, Blood Glucose and Triglyceride Levels in Sprague Dawley Rats
}

\author{
Jenalynne Mareah O. Chua, ${ }^{1}$ Deo Al Patrick T. Alonso, ${ }^{1}$ Elise Angela H. Espinosa, ${ }^{1}$ \\ Aya Isabel B. Gelonga, ${ }^{1}$ Abegail Visia Marie C. Silang ${ }^{1}$ and Ronald G. Garvilles ${ }^{2}$ \\ ${ }^{1}$ College of Public Health, University of the Philippines Manila \\ ${ }^{2}$ Department of Nutrition, College of Public Health, University of the Philippines Manila
}

\begin{abstract}
Background. Rice is the staple food in the Philippines, and the major carbohydrate source that greatly affect the weight, blood glucose and triglyceride levels of the population.
\end{abstract}

Objective. The objective of the researchers was to determine the effects of white, brown and red rice varieties on weight, blood glucose and triglyceride levels in rats

Methods. Sprague Dawley rats were fed with diets of cooked rice of different rice variety; white, brown and red. Weight was measured using a digital laboratory weighing scale. Blood glucose and triglyceride levels were measured by glucometer (OneTouch $\left.{ }^{\circledR}\right)$ and Humalyzer Primus ${ }^{\circledR}$, respectively.

Results. Rats fed with white rice had the greatest increase in weight and blood glucose levels, and red rice had the least increase. For the postprandial blood glucose test, all three rice groups reached their peak blood glucose level at 30 minutes, with white rice having the greatest value, followed by brown rice, and lastly, red rice. Rats fed with red rice had the greatest decrease in triglyceride level.

Conclusion. In this study, consumption of white rice contributes to weight gain, blood glucose level increase and minimal triglyceride level change compared to brown and red rice.

Key Words: rice, blood glucose, postprandial blood glucose, triglyceride, weight

\section{INTRODUCTION}

The Philippines is one of the world's emerging diabetes hotspots. Ranked in the top 15 in the world for diabetes prevalence, the Philippines is home to about 3.5 million people diagnosed with the disease in $2015 .{ }^{1}$ It is a public health issue to monitor nationwide prevalence of diabetes as it leads to significant cardiovascular-related mortality as well as significant complications such as End Stage Renal Disease (ESRD), blindness and lower leg amputations. ${ }^{2}$

A study by the Food and Nutrition Research Institute (FNRI) in 2011 showed that $22.3 \%$ of Filipino adults are overweight and $6.1 \%$ are obese. ${ }^{3}$ Additionally, an increase in the prevalence of atherosclerotic risk factors such as hypertension, Diabetes Mellitus (DM), dyslipidemia and central obesity has been noted among Filipinos. In the $8^{\text {th }}$ National Nutritional Survey done by the FNRI, it was determined that in $2013,38.7 \%$ of the total population in the country has high triglyceride levels $(\geq 200 \mathrm{mg} / \mathrm{dL})$ and $5.6 \%$ of the population has high fasting blood glucose level $(\geq 126 \mathrm{mg} / \mathrm{dL}$ ). Records also show a $20.6 \%$ prevalence of 
hypertension among Filipinos aged 20 and older, and a 72\% prevalence of dyslipidemia. ${ }^{4}$ In addition, a study by the World Health Organization on non-communicable diseases in 2014 showed that $33 \%$ of deaths were due to cardiovascular diseases (CVD). ${ }^{5}$

On the other hand, rice is known to be a good source of carbohydrates. Furthermore, it is also the leading source of sustenance among Filipinos. Total rice consumption of Filipinos has generally increased over time. This is because rice is considered as the staple food for Filipinos' diet, being their major carbohydrate source. Filipinos spend more on rice than on any other food and are now depending solely on rice more than ever for their energy supply and dietary protein as it remains as the most affordable food in the country. Furthermore, $47 \%$ of an average Filipino's daily caloric intake consist of rice. ${ }^{6}$ Carbohydrates affect blood glucose. Moreover, as the level of consumption of carbohydrates increases, blood concentrations of triglycerides also increases. ${ }^{7}$

DM and CVD are prevalent health conditions in the Philippines and these conditions are affected by several risk factors; two of which are elevated levels of blood glucose and triglyceride. The varieties of rice consumed by the Filipinos may have different effects on their blood glucose and triglycerides. Evidently, there is a need to study different carbohydrate sources and its relationship with blood glucose and triglyceride levels.

Since the three rice varieties, white, brown and red rice are staple food in agricultural countries such as the Philippines, they are among the major and dominant carbohydrate sources that greatly affect the weight, blood glucose and triglyceride levels of the population. ${ }^{6,8}$ Since the rice varieties differ in their composition, their individual effects on these two measurements differ as well.

Proper dietary management is of utmost importance in the prevention of occurrences of diabetes and other noncommunicable diseases linked to poor diet management. Thus, there is a need for identification of rice varieties that either have low glycemic index or have special antidiabetic characteristics in order to aid in the control and management of glycemic levels in those at risk of diabetes and those who already have diabetes.

Several studies, such as the studies by Yumiko Yoshizaki et al. and Denardin et al., utilized rodents to test the effects of different colored rice varieties on several parameters such as weight, blood glucose, triglyceride levels, and postprandial blood glucose. ${ }^{9,10}$ In both studies, the rodents had undergone a period of acclimatization before progressing to the experimental phase. The rodents were assigned to groups of the chosen rice varieties, were fed with the respective rice varieties and by the end of the experiment, the aforementioned parameters were measured and compared across the groups.

However, there have been limited studies, especially in the Philippines, that investigate and compare the glucometabolic response of people who eat red rice versus people who eat brown rice, and this comparison was evaluated in this study as a preliminary and exploratory research. It is also important to note that local red rice varieties also have not undergone such studies.

In line with this, the objective of study was to determine the effects of different rice varieties on the weight, blood glucose, and triglyceride levels in rats.

\section{METHODS}

\section{Design}

This research followed an analytic experimental design, particularly, randomized control trials. The sampling size consisted of 30 male rats that was divided and assigned into three exposure groups by randomization. The sample population for this study consisted 21-day old (weanling) Sprague Dawley rats. All rats were in-bred and having similar weights. The sample did not include rats with any pathologic or other abnormal conditions. The rats were procured from the Food and Drug Administration Philippines.

The rats were fed with a modified diet which consists $29.829 \%$ of commercially prepared, powdered non-fat milk, $12.427 \%$ of coconut oil, $56.744 \%$ of the cooked rice, and $1 \%$ vitamin and mineral complex by weight. ${ }^{11} \mathrm{~A}$ commercially prepared vitamin and mineral complex was used and the composition of it as follows: 1000 IU beta-carotene, 1500 IU retinol acetate, 500 IU cholecalciferol, 50 IU tocopheryl acetate, $30 \mathrm{mcg}$ phytomenadione, $60 \mathrm{mg}$ ascorbic acid, $1.5 \mathrm{mg}$ thiamine mononitrate, $1.7 \mathrm{mg}$ riboflavin, $20 \mathrm{mg}$ niacinamide, $3 \mathrm{mg}$ pyridoxine $\mathrm{HCl}, 400 \mathrm{mcg}$ folic acid, 25 mcg cyanocobalamin, $30 \mathrm{mcg}$ biotin, $50 \mathrm{mg}$ magnesium, 11 $\mathrm{mg}$ zinc, $55 \mathrm{mcg}$ selenium, $0.5 \mathrm{mg}$ copper, $2.3 \mathrm{mg}$ manganese, $45 \mathrm{mcg}$ chromium, $45 \mathrm{mcg}$ molybdenum, $72 \mathrm{mg}$ chloride, 80 $\mathrm{mg}$ potassium, $2 \mathrm{mg}$ silicon, $10 \mathrm{mcg}$ vanadium, $5 \mathrm{mcg}$ nickel, $150 \mathrm{mcg}$ boron, $250 \mathrm{mcg}$ lutein, and $300 \mathrm{mcg}$ lycopene. The white, brown and red rice were cooked with a proportion of 1,2 and 2.5 cups of water per 1 cup of uncooked rice respectively. The rice was cooked using a rice cooker for about $25-30$ minutes.

It is also important to note that diets were not isocaloric during the experimental process.

The rats underwent 5 days of acclimatization in which the rats were fed with commercially available rodent feeds, followed by 25 days of experimental phase in which they were fed with the experimental diet based on the rice variety assigned to their respective group. At the end of the acclimatization phase, baseline measurements were taken.

Baseline measures were established for the three variables of interest. Weight was measured using a calibrated analytical balance. Blood glucose level was measured using the OneTouch ${ }^{\circledR}$ Select Simple Blood Glucose Monitoring System. Triglyceride level was measured spectrophotometrically using the HumalyzerPrimus ${ }^{\circledR}$ which was provided by the University of the Philippines Manila, College of Public Health's nutrition laboratory. The triglyceride reagent kit used was a Triglycerides Mono 
Kit $(4 \times 100)$ procured from HUMAN Gesellschaft für Biochemica und Diagnostica mbH. For the measurement of the baseline levels, blood was obtained from the periorbital region using a capillary tube, as suggested by the Institutional Animal Care and Use Committee (IACUC).

After the experimental phase, the parameters were measured once again for the final values. Blood for the final measurement was obtained through cardiac puncture.

Postprandial blood glucose was also analyzed in order to determine and compare the sudden rise in blood glucose level of the rats after consuming a meal. Blood was extracted from a small incision with a scalpel blade hitting the saphenous vein, and postprandial glucose was monitored 15,30 , and 60 minutes after the consumption of the meal. This was done on the 14th day of the experimental phase. Rats have increased possibility of dying with multiple blood extraction that causes increased stress. Multiple blood extraction until 120 minutes is usually done only on humans. If this were to be done to rats, it would be too stressful for them. Thus, blood was extracted for four times only per rat for the postprandial blood glucose test.

At the end of the experimental phase, the rats were anesthetized through intramuscular injection of Zoletil ${ }^{\circledR}$, a mixture of tiletamine and zolazepam, following a dosage of $50-75 \mathrm{mg}$ per $\mathrm{kg}$ body weight for rats. Midventral puncture was done to collect blood for analysis of blood glucose and triglyceride levels, because this is where the pulse is easily located. The rats were then euthanized by cervical dislocation. This was done under the supervision of faculty members of the Department of Nutrition, College of Public Health, University of the Philippines-Manila and a licensed veterinarian. Carcasses were properly disposed of following the appropriate protocol.

\section{Statistical Analysis}

Mean values of the differences of the initial and final weight, blood glucose level, and triglyceride level were analyzed using one-way ANOVA and Bonferroni's Post Hoc Test. One-way ANOVA is used to determine whether the mean of a dependent variable is the same in two or more unrelated independent groups. In this study, the independent variable was the rice variety. The independent variable yielded three independent groups namely the White Rice Group, Brown Rice Group, and the Red Rice Group. On the other hand, the three parameters were the dependent variables (weight, blood glucose levels, and triglyceride). The mean results per parameter were computed per group and the resulting $p$-value indicated the presence of any significant difference of the parameter among the three groups. However, the $p$-value does not indicate where the significant differences lie among the three groups (e.g. which rice variety specifically yielded a significant result), therefore there was a need to use the Bonferroni's Post Hoc test.

On the other hand, results from the postprandial blood glucose test were subjected to two-way repeated measures
ANOVA to determine whether there was a significant difference between the effects of rice varieties on the glucose measurements across time and whether rice varieties interacted with time.

Statistical analysis was performed with Stata ${ }^{\circledR}$. The study was worked with the $\alpha$-significance level of 0.05 .

\section{RESULTS AND DISCUSSION}

\section{Weight}

The results of the study indicated that rats from the white rice group showed the greatest increase in mean weight among the three rice groups, followed by rats from the brown rice group, and lastly from the red rice group (Tables 1 and 4). The weights of all the rats were measured within the whole course of experimental phase for 7 days on Days 1, 4, 7, 9,13,21, and 23. The mean weights per day were plotted in a line graph (Figure 1).

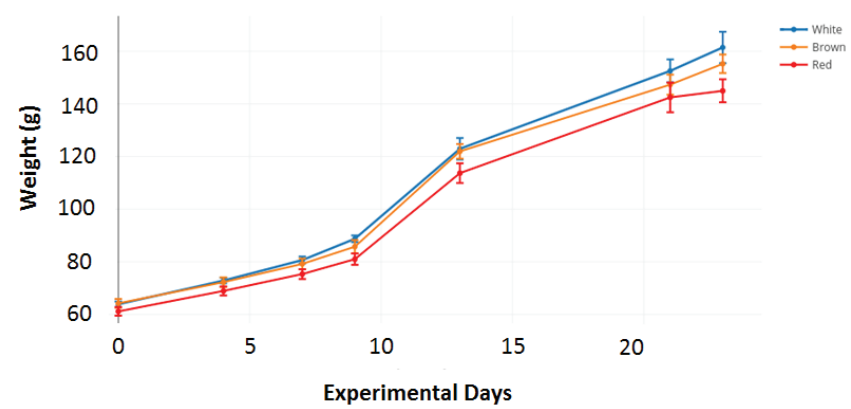

Figure 1. Mean experimental weights of the rats from all rice groups on days 1, 4, 7, 9, 13, 21, and 23. Each value represents the mean \pm SE. Legend: Blue line represents values for the White Rice Group. Orange line represents the values for the Brown Rice Group. Red line represents the values for the Red Rice Group.

This trend may be further explained by the differences in the composition of the three rice varieties. White rice is produced upon the milling of brown rice where bran layers are removed. Along with these bran layers, fiber-rich phytochemicals are removed as well.

These phytochemicals decrease fat mass and increase satiety by stimulating digestion and enhancing the activity of enzymes needed for digestion. ${ }^{12}$ Brown rice also contains $\gamma$-oryzanol which inhibits the activity of hypothalamic endoplasmic reticulum stress-responsive genes, thereby attenuating the preference of rats for high fat diet. ${ }^{13}$ As for red rice, they are known to be rich in phenolic acid content and flavonoids which essentially indicates low glycemic index indicating a slower rate of glucose absorption, ${ }^{14}$ prompting a more gradual rise in blood glucose level, and slowing down gastric emptying. ${ }^{15}$ These factors all contribute to the lower mean weight difference in brown and red rice compared to white rice.

However, with the $p$-value obtained after statistical analysis as seen in Table 4, it is still important to note that 
Table 1. Initial, final and the change in the weight (g) of the rats belonging to three rice groups

\begin{tabular}{|c|c|c|c|c|c|c|c|c|c|}
\hline \multirow{2}{*}{ Rat } & \multicolumn{3}{|c|}{ White Rice Group } & \multicolumn{3}{|c|}{ Brown Rice Group } & \multicolumn{3}{|c|}{ Red Rice Group } \\
\hline & Initial & Final & Change & Initial & Final & Change & Initial & Final & Change \\
\hline 1 & 63.0 & 175.8 & 112.8 & 60.4 & 153.9 & 93.5 & 69.0 & 160.2 & 91.2 \\
\hline 2 & 67.9 & 170.1 & 102.2 & 66.4 & 153.4 & 87.0 & 52.6 & 125.9 & 73.3 \\
\hline 3 & 63.8 & 114.1 & 50.3 & 70.2 & 162.2 & 92.0 & 59.2 & 157.7 & 98.5 \\
\hline 4 & 61.9 & 163.9 & 102.0 & 65.8 & 171.0 & 105.2 & 57.4 & 152.9 & 95.5 \\
\hline 5 & 61.8 & 168.6 & 106.8 & 73.2 & 164.5 & 91.3 & 59.8 & 138.9 & 79.1 \\
\hline 6 & 60.9 & 167.5 & 106.6 & 62.8 & 156.4 & 93.6 & 65.9 & 143.6 & 77.7 \\
\hline 7 & 70.2 & 177.9 & 107.7 & 54.3 & 130.1 & 75.8 & 56.8 & 148.8 & 92.0 \\
\hline 8 & 65.5 & 160.3 & 94.8 & 62.2 & 156.3 & 94.1 & 62.7 & 162.5 & 99.8 \\
\hline 9 & 59.3 & 146.3 & 87.0 & 66.4 & 145.8 & 79.4 & 67.2 & 124.5 & 57.3 \\
\hline 10 & 64.8 & 169.9 & 105.1 & 60.8 & 158.5 & 97.7 & 61.2 & 134.5 & 73.3 \\
\hline Ave. & 64.8 & 169.9 & 97.53 & 60.8 & 158.5 & 90.96 & 61.2 & 134.5 & 83.77 \\
\hline
\end{tabular}

Table 2. Initial, final and the change in the blood glucose levels ( $\mathrm{mg} / \mathrm{dl}$ ) of the rats belonging to three rice groups

\begin{tabular}{|c|c|c|c|c|c|c|c|c|c|}
\hline \multirow{2}{*}{ Rat } & \multicolumn{3}{|c|}{ White Rice Group } & \multicolumn{3}{|c|}{ Brown Rice Group } & \multicolumn{3}{|c|}{ Red Rice Group } \\
\hline & Initial & Final & Change & Initial & Final & Change & Initial & Final & Change \\
\hline 1 & 130.5 & 233.5 & 103.00 & 70.0 & 195.5 & 125.50 & 73.0 & 142.0 & 69.00 \\
\hline 2 & 101.0 & 232.5 & 131.50 & 104.0 & 309.5 & 205.50 & 128.5 & 81.5 & (-) 47.00 \\
\hline 3 & 81.0 & 217 & 136.00 & 97.5 & 173.0 & 75.50 & 77.0 & 153.5 & 76.50 \\
\hline 4 & 81.0 & 247.5 & 166.50 & 81.0 & 151.5 & 70.50 & 64.5 & 115.5 & 51.00 \\
\hline 5 & 99.5 & 255.5 & 156.00 & 140.5 & 147.5 & 7.00 & 93.0 & 92.0 & (-) 1.00 \\
\hline 6 & 80.5 & 133 & 52.50 & 79.5 & 166.0 & 86.50 & 75.0 & 130.5 & 55.50 \\
\hline 7 & 114.5 & 204.5 & 90.00 & 70.0 & 110.0 & 40.00 & 74.5 & 109.0 & 34.50 \\
\hline 8 & 84.5 & 114 & 29.50 & 84.0 & 127.5 & 43.50 & 90.0 & 206.0 & 116.00 \\
\hline 9 & 102.5 & 136 & 33.50 & 98.0 & 119.5 & 21.50 & 101.0 & 126.0 & 25.00 \\
\hline 10 & 140.0 & 219 & 79.00 & 86.0 & 197.0 & 111.00 & 108.0 & 156.0 & 48.00 \\
\hline Ave. & 101.5 & 199.25 & 97.75 & 91.05 & 169.7 & 78.65 & 88.45 & 131.2 & 42.75 \\
\hline
\end{tabular}

Table 3. Initial, final and the change in the triglyceride levels $(\mathrm{mg} / \mathrm{dl})$ of the rats belonging to three rice groups

\begin{tabular}{|c|c|c|c|c|c|c|c|c|c|}
\hline \multirow{2}{*}{ Rat } & \multicolumn{3}{|c|}{ White Rice Group } & \multicolumn{3}{|c|}{ Brown Rice Group } & \multicolumn{3}{|c|}{ Red Rice Group } \\
\hline & Initial & Final & Change & Initial & Final & Change & Initial & Final & Change \\
\hline 1 & N/A & 31.00 & $N / A$ & $\mathrm{~N} / \mathrm{A}$ & 16.14 & $\mathrm{~N} / \mathrm{A}$ & $\mathrm{N} / \mathrm{A}$ & 82.87 & $\mathrm{~N} / \mathrm{A}$ \\
\hline 2 & $\mathrm{~N} / \mathrm{A}$ & 45.27 & $N / A$ & 78.93 & 32.28 & (-) 46.65 & N/A & 85.03 & N/A \\
\hline 3 & N/A & 25.29 & $N / A$ & 86.02 & 26.67 & (-) 59.35 & N/A & 35.72 & $\mathrm{~N} / \mathrm{A}$ \\
\hline 4 & N/A & 84.93 & $\mathrm{~N} / \mathrm{A}$ & 125.8 & 93.79 & (-) 31.98 & 61.61 & 93.99 & 32.38 \\
\hline 5 & 80.80 & 133.70 & 52.90 & 48.13 & 84.54 & 36.41 & 40.15 & 42.42 & 2.270 \\
\hline 6 & 161.89 & 52.06 & (-) 109.8 & 55.11 & 78.05 & 22.94 & 73.02 & 56.00 & (-) 17.02 \\
\hline 7 & 53.93 & 71.74 & 17.81 & 130.8 & 100.8 & (-) 30.01 & N/A & 41.73 & N/A \\
\hline 8 & $\mathrm{~N} / \mathrm{A}$ & 51.47 & $\mathrm{~N} / \mathrm{A}$ & 147.3 & 103.8 & (-) 43.55 & 174.3 & 79.52 & (-) 94.77 \\
\hline 9 & 46.16 & 77.45 & 31.29 & 84.34 & 74.50 & (-) 9.840 & 79.32 & 56.59 & (-) 22.73 \\
\hline 10 & 153.82 & 57.82 & (-) 96.00 & $\mathrm{~N} / \mathrm{A}$ & 40.25 & N/A & 138.6 & 45.76 & (-) 92.81 \\
\hline Ave. & 99.32 & 63.07 & (-) 20.77 & 94.55 & 65.08 & (-) 20.25 & 94.49 & 61.96 & $(-) 32.11$ \\
\hline
\end{tabular}

Table 4. Mean differences in the initial and final measurements for weight, blood glucose, and triglyceride levels in rats belonging to white, brown and red rice groups

\begin{tabular}{cccc}
\hline Rice Variety & Weight & Blood Glucose Level & Triglyceride Level \\
White & $97.53 \pm 5.73 \mathrm{~g}$ & $97.75 \pm 15.63 \mathrm{mg} / \mathrm{dL}$ & $-20.77 \pm 24.09 \mathrm{mg} / \mathrm{dL}$ \\
Brown & $90.96 \pm 2.69 \mathrm{~g}$ & $78.65 \pm 18.44 \mathrm{mg} / \mathrm{dL}$ & $-20.25 \pm 10.81 \mathrm{mg} / \mathrm{dL}$ \\
Red & $83.77 \pm 4.36 \mathrm{~g}$ & $42.75 \pm 14.07 \mathrm{mg} / \mathrm{dL}$ & $-32.11 \pm 16.29 \mathrm{mg} / \mathrm{dL}$ \\
\hline$p$-value & 0.1093 & 0.0671 & 0.9053 \\
\hline
\end{tabular}

there is no sufficient evidence to support the effects of different rice varieties on the weight of Sprague Dawley Rats.

\section{Blood Glucose}

In this study, rats from the white rice group exhibited the greatest increase in blood glucose levels by the end of the study, while red rice group exhibited the least increase (Tables
2 and 4). This is further supported by previous studies where red rice was shown to possess anti-diabetic related properties such as anti-hyperglycemic action for the management of diabetes and its underlying consequences. ${ }^{16}$ Pigmented rice varieties like red rice are also rich in phenolic acid, with vanillic acid as the most abundant type, and flavonoid content, with tricin as the most abundant type, contributing 
to the increased activity of the enzyme glucokinase. This enzyme consequently functions to phosphorylate glucose to glucose-6-phosphate, which is the first step in glycogen synthesis in the liver. As a result, there is decrease in blood glucose levels due to the increase in glucose uptake. ${ }^{14}$

The analysis of data in Table 4 indicated lower increase in blood glucose for both red and brown rice compared to white rice, but results showed no significant difference in between the three treatment groups, with a $p$-value of 0.0671 . However, the $p$-value was close to borderline significance. Therefore, there is no sufficient evidence to support the effect of different rice varieties on the blood glucose levels of Sprague Dawley Rats.

\section{Post-Prandial Blood Glucose}

The post-prandial blood glucose level test is used to determine the amount of glucose present in the blood right after a meal as explained previously in the methods. Blood was extracted from a small incision on the saphenous vein.

Means of the three groups reached their peak blood glucose level at 30 minutes, with white rice having the greatest value, followed by brown rice, and lastly, red rice. The trend of the blood glucose levels across the time of 0, 15, 30 and 60 minutes (Figure 2).

However, there was no sufficient evidence to conclude the significance of the interaction between time and rice varieties

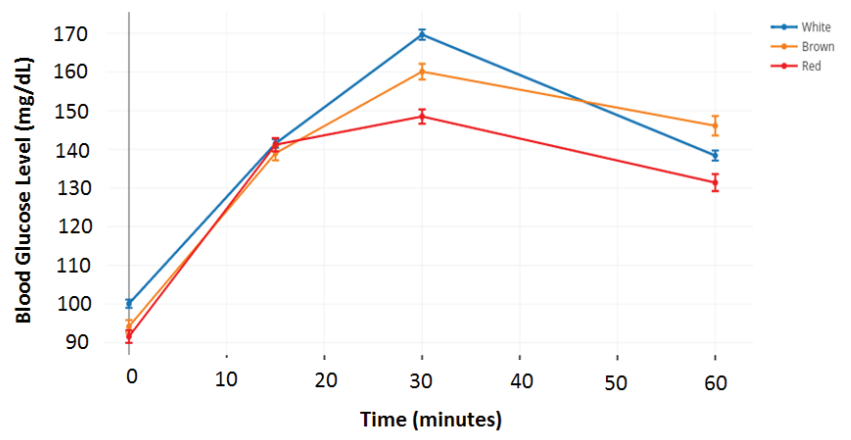

Figure 2. Post-prandial Blood Glucose. Mean blood glucose levels at $0,15,30$, and 60 minutes of the rats from the three rice groups. Each value represents the mean \pm SE.

Legend: Blue line represents values for the White Rice Group. Orange line represents the values for the Brown Rice Group. Red line represents the values for the Red Rice Group.

based on the $p$-value of 0.7016 obtained. Likewise, there is no sufficient evidence to conclude significant interaction between rice varieties with the $p$-value of 0.5490 .

Glycemic index or GI is a measure that indicates the rate by which blood glucose will rise in response to foods containing carbohydrate ${ }^{17}$ According to related literature, white rice has an average GI of $72,{ }^{18}$ while brown and red rice have average GIs of 55 and $50^{19}$ respectively. Food is usually considered to have a high glycemic index when their values fall $\geq 70$. It is considered intermediate if glycemic index is between $56-69$ and low if glycemic indices have values that fall $\leq 55$. Thus, white rice is a high GI food while brown and red rice are classified as low GI. Food having a relatively higher GI would cause a spike in the blood glucose levels after eating. This is because of the different components found in rice varieties. White rice, having a high glycemic index, can lead to a rapid rise and subsequent rapid fall of blood glucose after its peak. Due to the high fat and fiber content of brown rice, the rise and fall in blood glucose is rather gradual compared to the white rice. ${ }^{20}$ The same gradual rise and fall of glucose concentration for the rats fed with red rice is due to the high phenolic and flavonoid content of red rice. ${ }^{14}$

\section{Triglyceride}

Similar to the parameters mentioned earlier, in this study, rats from the white rice group exhibited the least decrease in triglyceride levels by the end of the study, while red rice exhibited the greatest decrease (Tables 3 and 4).

The red rice's ability to suppress the increase triglyceride levels is in line with a study in which hepatic and serum triglycerides were found to be lower in mice fed with red rice in comparison to those fed with brown rice, due to its high antioxidant and phenolic compound content, specifically phenolic acids, flavonoids and anthocyanins, with vanillic acid, tricin, and cyaniding-3-O-glucoside as the most abundant type from each of the 3 groups respectively, containing $54.8 \mathrm{mg}$ vanillic acid, $101.7 \mathrm{mg}$ tricin, and $700.2 \mathrm{mg}$ cyaniding-3-Oglucoside, per $100 \mathrm{~g}$ of rice. ${ }^{14,21}$ This is further supported by a previous study where phenolic acid-rich extract administration resulted to significant suppression of the elevation of blood and liver concentrations of triglyceride. It should also be noted that cooking may have affected the results as well. In a previous study, it was shown that hydrothermal process increased the $\gamma$-oryzanol concentrations in brown rice and decreased the said concentrations in red rice, thus narrowing down the difference between the two rice varieties. ${ }^{22}$ In this study, the hydrothermal process on this study is done through wet-cooking, specifically, steaming of rice.

\section{CONCLUSION AND RECOMMENDATIONS}

The results obtained from this study indicate that there are indeed trends in the effects of the rice varieties to the three parameters of interest-favoring brown and red rice rather than white. However, it is essential to reiterate that despite the observed trends, this study showed no sufficient evidence of significant differences in weight among Sprague Dawley rats due to them being fed different rice varieties. It also did not statistically demonstrate significant changes in blood glucose and triglyceride levels of the Sprague Dawley rats.

The researchers recommend the use of an isocaloric diet. Isocaloric diet is recommended all throughout the future experiments in order to observe the effects of other components (besides the carbohydrates, fats and protein) of the rice varieties on the three variables of interest in the study. 
Since GI measurements found in related literature are based on GI tests done on human subjects, the GI may not be exactly reflective of the supposed GI of rice varieties if generated from rats. GI tests may be performed on rats for future studies.

The insulin levels in the blood after ingestion of the rice varieties (postprandial) may also be measured. This is to determine whether the rice varieties and the active components present affect insulin levels as well. The interaction between blood glucose and insulin may also be evaluated.

Further examination of liver and adipose tissue is also recommended to histologically visualize the fat content of the rat hepatic cells.

Future experiments may include nutritional analysis of the rice to be tested. This way, the components and active substances in the rice will be properly quantified and described. Consequently, the effects of the said substances may be measured and observed individually.

Other rice varieties may also be considered for alternative feed. The varieties used in the study were white, brown, and red rice. Analysis of daily water intake is suggested in order to account for its effects on the food intake and weight of the rats. It is also recommended to increase the duration of the experimental phase as done by other researchers with similar studies. Increasing the sample size is also recommended.

Lastly, computation of Area Under Curve is recommended as it is the most appropriate method of analysis for post-prandial blood glucose test.

\section{Acknowledgments}

The study was funded by the Research Grants Administration Office (RGAO) of the University of the Philippines-Manila.

The authors acknowledge the support of Dr. Rohani Cena and Dr. Alice Alma Bungay of the Institutional Animal Care and Use Committee (IACUC) for thoroughly reviewing our protocol. We would also like to thank Mr. Meliton Balighot of National Institutes of Health (NIH), for assisting us in our blood extraction procedures.

\section{Statement of Authorship}

All authors have approved the final version submitted.

\section{Author Disclosure}

All authors have declared no conflict of interest.

\section{Funding Source}

The project was fully funded by University of the Philippines Manila Student Research Grant.

\section{REFERENCES}

1. Diabetes: facts and figures, International Diabetes Federation [Online]. 2016 [cited 2016 Sept]. Available from http://www.idf.org/aboutdiabetes/facts-figures.
2. Jimeno CA, Kho SA, Matawaran BJ, Duante CA, Jasul GV. Prevalence of diabetes mellitus and pre-diabetes in the Philippines: a sub-study of the 7th National Nutrition and Health survey. Philipp J Intern Med. 2015; 53 (2):1-8.

3. 8th National Nutrition Survey, FNRI Website [Online]. 2016 [cited 2016 Sept]. Available from http://www.fnri.dost.gov.ph/index.php/ nutrition-statistic/19-nutrition-statistic/118-8th-national-nutritionsurvey.

4. Philippines leads countries with highest obesity levels, Philippine Star website [Online]. 2014 [cited 2016 Sept]. Available from http://www. philstar.com/headlines/2014/10/20/1382165/phl-leads-countrieshighest-obesity-levels.

5. World Health Organization. Noncommunicable diseases country profiles. 2014.Geneva, Switzerland: WHO Document Production Services. 2014;148

6. Santiaguel AF. Nourishing a nation. Rice Today- International Rice Research Institute. 2013; 12 (3):11.

7. Parks EJ. Effects of dietary carbohydrate on triglyceride metabolism in humans. J Nutr. 2001; 131(10):2772S-4S.

8. GRiSP (Global Rice Science Partnership). Rice almanac, 4th edition. Los Baños (Philippines): International Rice Research Institute. 2013. pp. 139-43

9. Yoshizaki Y, Kawasaki C, Cheng KC, et al. Rice koji reduced body weight gain, fat accumulation, and blood glucose level in high-fat dietinduced obese mice. PeerJ. 2014; 2:e540. doi: 10.7717/peerj.540.

10. Denardin CC, Boufleur N, Reckziegel P, Silva LPD, Walter M. Amylose content in rice (Oryza sativa) affects performance, glycemic and lipidic metabolism in rats. Cienc Rural. 2012; 42(2):381-7.

11. Laboratory Rodent Diet, www.labdiet.com [Online]. 2015 [cited 2016 Sept]. Available from http://www.labdiet.com/cs/groups/lolweb/@ labdiet/documents/web_content/mdrf/mdi4/ edisp/ducm04_028021. pdf

12. Kim JY, Kim JH, Lee DH, Kim SH, Lee SS. Meal replacement with mixed rice is more effective than white rice in weight control, while improving antioxidant enzyme activity in obese women. Nutr Res. 2008; 28(2):66-71.

13. Kozuka C, Yabiku K, Sunagawa S, et al. Brown rice and its component, $\gamma$-oryzanol, attenuate the preference for high-fat diet by decreasing hypothalamic endoplasmic reticulum stress in mice. Diabetes. 2012; 61(12):3084-93 doi: 10.2337/db11-1767. Epub 2012 Jul 23

14. Boue SM, Daigle KW, Chen MH, Cao H, Heiman M. Antidiabetic potential of purple and red rice (Oryza sativa L.) bran extracts. J Agric Food Chem. 2016; 64(26):5345-53.

15. Jenkins DJA, Kendall CWC, Augustin LSA, et al. Glycemic index: overview of implications in health and disease. Am J Clin Nutr 2002; 76(suppl):266S-73S.

16. Abeysekera WKSM, Arachchige SPG, Ratnasooriya WD, Choudhary MI, Dalvandi K, Chandrasekharan NV. Anti-diabetic related health food properties of traditional rice (Oryza sativa L.) in Sri Lanka. 2015; 3(10): 815-20.

17. Hettiarachchi P, Jiffry MT, Jansz ER, Wickramasinghe AR, Fernando DJ. Glycaemic indices of different varieties of rice grown in Sri Lanka. Ceylon Med J. 2001;46(1):11-4.

18. Publications H. Glycemic index and glycemic load for 100+ foods, Harvard Health [Online]. 2017 [cited 2017 March]. Available from http://www.health.harvard.edu/diseasesconditions/glycemic_index_ and_glycemic_load_for_100_foods

19. Carbohydrates and the Glycemic Index, Webnat.com [Online]. 2017 [cited 2017 March]. Available from https://www.webnat.com/articles/ Glycemix.asp.

20. Juliano, B. Nutritional value of rice and rice diets. In: Juliano B eds Rice in human nutrition. FAO Food and Nutrition Series; 1993. pp. 61-84.

21. Park Y, Park EM, Kim EH, Chung IM. Hypocholesterolemic metabolism of dietary red pericarp glutinous rice rich in phenolic compounds in mice fed a high cholesterol diet. Nutr Res Pract. 2014; 8(6):632-7.

22. Min B, McClung A, Chen M. Effects of hydrothermal processes on antioxidants in brown, purple and red bran whole grain rice (Oryza sativa L.). Food Chem. 2014; 159:106-15. 